\title{
Reisestipendium 2020 der Deutschen AO
}

Karl-Heinz Frosch

\section{Anträge auf das AOTrauma Deutschland-Reisestipendium 2020 können bis Freitag, 31.01.2020 eingereicht werden.}

Die AOTrauma Deutschland vergibt jährlich mindestens ein Reisestipendium zur Förderung des Erfahrungsaustausches und der Weiterbildung bei angesehenen Experten in unserer Disziplin. Der Maximalbetrag pro Stipendium beträgt 5000,- EUR. Bewerben können sich klinisch aktive Mitglieder der deutschen AOTrauma, AOCMF und AOVET (Eintritt ist bis Bewerbungsfristende möglich). Einzureichen sind ein Motivationsschreiben (max. 1 Seite), Name der zu besuchenden Klinik und des Arztes sowie ein Kurz-CV. Nach Abschluss der Reise ist ein kurzer Bericht zu schreiben. Eine Auswahlkommission wird die Entscheidung treffen.

Anträge können eingereicht werden bis zum 31. Januar (Poststempel oder E-Mail-Eingang vor Mitternacht am 31.01.2020) bei:
Univ.-Prof. Dr. Karl-Heinz Frosch

AOTD Community Development Officer

Klinik für Unfall, Hand- und Wiederherstellungschirurgie Universitätsklinikum Hamburg-Eppendorf Martinistraße 52

20246 Hamburg

E-Mail: k.frosch@uke.de

Prof. Dr. Karl-Heinz Frosch, UKE, Hamburg

Bibliografie

DOI https://doi.org/10.1055/a-1028-7907 OP-JOURNAL 2019; 35: 353 ๑ Georg Thieme Verlag KG Stuttgart · New York ISSN 0178-1715 